
FIELDS

\title{
SOLITARY-WAVE AND PERIODIC SOLUTIONS OF THE KURAMOTO-VELARDE DISPERSIVE EQUATION
}

\author{
OGNYAN Y. KAMENOV \\ Department of Applied Mathematics and Informatics, \\ Technical University of Sofia, P.O. Box 384, 1000 Sofia, Bulgaria, \\ e-mail: okam@abv.bg
}

[Received 01 February 2016. Accepted 29 February 2016]

\begin{abstract}
In the present paper, solitary solutions of the KuramotoVelarde (K-V) dispersive equation have been found, using the deformation and mapping approach. These exact solutions show the dynamics and the evolution of dispersive solitary waves. In the case $\alpha_{2}=\alpha_{3}$, three families of exact periodic solutions have been obtained by employing the bilinear transformation method.

KEY WORDS: Solitary-wave solutions, periodic solutions, non-integrable and semi-integrable evolution equations.

Mathematics Subject Classification: 35G20.
\end{abstract}

\section{Introduction}

The nonlinear evolution dispersive equation $\mathrm{K}-\mathrm{V}$

$$
u_{t}+\alpha_{0}\left(u_{x x}+u_{x x x x}\right)+\alpha_{1} u_{x x x}+\alpha_{2} u_{x}^{2}+\alpha_{3} u u_{x x}=0,
$$

where $u$ is a real-valued function, $\alpha_{j} \in \mathbb{R}, j=0, \ldots 3, \alpha_{0} \geq 0, \alpha_{1} \neq 0, \alpha_{2} \neq 0$, $\operatorname{sign} \alpha_{2}=\operatorname{sign} \alpha_{3}$, is a generalization of the Kuramoto-Velarde (KV) equation corresponding to the case $\alpha_{1}=0$ and of the dispersive Kuramoto-Sivashinsky (KS) equation which corresponds to the case $\alpha_{3}=0$. The dispersive (KS) equation is a model equation for long waves on a viscous fluid flowing down an inclined plane [1], as well as for drift waves in plasma [2]. The KV equation is an equation describing slow space-time variations of disturbances at interfaces, diffusion-reaction fronts and plasma instability fronts [3] and [4]. The dispersive $\mathrm{K}-\mathrm{V}$ equation combines in its linear part dispersive and dissipative effects. The 
numerical and asymptotic analyses of the nonlinear evolution KV equation show the typical bifurcation diagram of solutions [5], due to the nonlinear terms.

The present work is focused on finding the exact localized solitary and periodic solutions, in the second and third sections, respectively, which have not been obtained up to now.

\section{Exact solitary solutions}

Instead of equation (1) it is more convenient to use the equation

$$
u_{t}+u_{x x}+\lambda u_{x x x}+\alpha u_{x x x x}+u_{x}^{2}+\mu u u_{x x}=0,
$$

which is an exact reduction of (1) under the rescaling

$$
t \rightarrow \frac{t}{\alpha_{0}^{3}} ; \quad x \rightarrow \frac{x}{\alpha_{0}} ; \quad u \rightarrow \frac{\alpha_{2} u}{\alpha_{0}} ; \quad \lambda=\alpha_{1} ; \quad \alpha=\alpha_{0}^{2} ; \quad \mu=\frac{\alpha_{3}}{\alpha_{2}} .
$$

We will prove the following theorem.

Theorem 1. If $\mu>1$ under the a priori conditions: $\lambda \neq 0, \alpha>0$, then the evolution equation (2) allows exact solitary-wave solution.

Proof. Considering the second degree of singularity of equation (2), we will seek its solution in the form

$$
u(x, t)=A \wp(\xi)+\frac{B \wp^{\prime}(\xi)}{\wp(\xi)-C}+E,
$$

where $A, B, C, E$ are real parameters, unknown at this stage, assuming that $C>0$, and $\wp(\xi)=\wp\left(\xi, G_{2}, G_{3}\right)$ is the basic elliptic Weierstrass function [6] with real invariants $G_{2}=12 C^{2}, G_{3}=-8 C^{3}$. The phase variable $\xi$ is defined by the equality $\xi=k x+k^{2} \omega t+\delta$, where in the general case $k, \omega, \delta$ are complex parameters, also unknown for the time being. Substituting (3) in equation (2), we will obtain the following algebraic system:

$$
\begin{aligned}
& 60 A k^{2} \alpha+A^{2}(3 \mu+2)=0 ; \quad 3 A+6 B k \lambda+2 B^{2}+\mu\left(3 A E+4 B^{2}\right)=0 ; \\
& B \omega-108 A C^{2} k^{2} \alpha+4 B^{2} C(\mu-1)-3 A^{2} C^{2}(\mu+2)=0 ; \\
& B C[\omega+6 C k \lambda+2 B C(4 \mu-1)]+A C^{2}(3+3 \mu E-4 A C)=48 A C^{3} k^{2} \alpha ; \\
& A \omega+2 B-4 A B C+2 B \mu(E+3 A C)=0 ; \\
& 3 A k \lambda+6 B k^{2} \alpha+A B(2 \mu+1)=0 .
\end{aligned}
$$


It is easy to comply that the system (4) is compatible since the third and the fourth equations are equivalent, so we could neglect the fourth equation.

$$
\begin{aligned}
& A=-\frac{60 \alpha k^{2}}{3 \mu+2} ; B= \pm \frac{30 k \lambda}{17 \mu+8} ; E=\frac{\lambda^{2}}{\alpha \mu}\left(\frac{3 \mu+2}{17 \mu+8}\right)^{2}-\frac{1}{\mu} \\
& \omega(k)=(\mu-1)\left[\frac{288 k^{3} \alpha^{2}(17 \mu+8)}{\lambda(3 \mu+2)^{2}} C^{2}+\frac{120 k \lambda}{(17 \mu+8)} C\right]
\end{aligned}
$$

It follows from the last equality that $\mu \neq 1$, because $\omega \neq 0$ a priori. The parameter $C$ is determined from the compatibility condition between the third and the fifth equation, i.e.

$$
\frac{3 A}{B}\left[36 k^{2} \alpha+A(\mu+2)\right] C^{2}+(2 \mu B) C+\frac{2 B}{A}(\mu E+1)=0 .
$$

The last equation determines two values for $C$ :

$$
C_{1}=\frac{1}{24}\left(\frac{\lambda}{k \alpha}\right)^{2}\left(\frac{3 \mu+2}{17 \mu+8}\right)^{2}\left(\frac{3 \mu+2}{\mu-1}\right), \quad C_{2}=\frac{1}{12}\left(\frac{\lambda}{k \alpha}\right)^{2}\left(\frac{3 \mu+2}{17 \mu+8}\right)^{2} .
$$

It is obvious that both values of $C$ are positive and real if $\mu>1$ under the a priori conditions for the rest of parameters.

If we assume that the phase shift $\delta$ is equal to the imaginary semi-period of the function $\wp\left(\xi, G_{2}, G_{3}\right)$, i.e.

$$
\delta=\frac{i K_{2}(m)}{\sqrt{e_{1}-e_{3}}}=\frac{i \pi}{2 \sqrt{3 C}}
$$

where $m=1, K_{2}(m)$ is the Legendre complete elliptic integral of second kind, as for $G_{2}=12 C^{2}, G_{3}=-8 C^{3}$ (i.e. $e_{1}=e_{2}=C, e_{3}=-2 C$ ), and also $K_{2}(1)=\pi / 2$. Using the phase modulation [6]

$$
\wp\left(\xi, 12 C^{2},-8 C^{3}\right)=C-3 C \operatorname{sech}^{2}(\theta \sqrt{3 C}), \theta=k(x+k \omega t),
$$

we can obtain the following one-parameter family of exact solitary-wave solutions of KVE:

$$
u(x, t)=3 A C \tanh ^{2}(\theta \sqrt{3 C}) \pm 2 B \sqrt{3 C} \tanh (\theta \sqrt{3 C})+(E-2 A C) .
$$

All parameters $A, B, C, E$ and $\omega$ are determined by the equality (5). The resulting exact, solitary-wave solution is of the type "shock wave". These 
waves would be bidirectional, since the wave number $k$ is a free positive parameter while the parameters $\lambda, \mu$ can be both positive and negative, on which depends the sign of $\omega(k, \lambda, \mu)$. The Theorem 1 has been proven by this.

\section{Periodic solution}

In the case when $\mu=1$ we will prove the following theorem.

Theorem 2. If $\mu=1$ the evolution equation (2) allows exact periodic solution.

Proof. Let us apply to equation (2) the Hirota-Satsuma transformation [7], assuming that $\mu=1$ (i.e. $\alpha_{2}=\alpha_{3}$ )

$$
u(x, t)=\beta_{0}+\beta_{1}(\ln \zeta)_{x x},
$$

where $\beta_{0}, \beta_{1}\left(\beta_{1} \neq 0\right)$ are unknown real parameters (at this stage), and $\zeta=$ $\zeta(x, t) \in C^{6}(\Omega), \Omega=\left\{(x, t) \in \mathbb{R}^{2},-\infty<x<\infty, 0<t<\infty\right\}$. After substituting (8) in (2) and a single integration with respect to $x$, we obtain the following bilinear representation (See Appendix A):

$$
\text { (9) } \begin{aligned}
\frac{1}{2 \zeta^{2}}\left(D_{t} D_{x} \zeta . \zeta+\lambda D_{x}^{4} \zeta . \zeta\right. & \left.+8 G \zeta^{2}\right)+\frac{\partial}{\partial x}\left\{\left(1+\beta_{0}\right) \frac{D_{x}^{2} \zeta . \zeta}{2 \zeta^{2}}+\alpha \frac{D_{x}^{4} \zeta . \zeta}{2 \zeta^{2}}+\right. \\
+ & \left.\left(\frac{\beta_{1}}{2}-6 \alpha\right)\left(\frac{D_{x}^{2} \zeta . \zeta}{2 \zeta^{2}}\right)^{2}+\left(H_{0}+\frac{\beta_{0}^{2}}{2 \beta_{1}}\right)\right\}=0
\end{aligned}
$$

where $G$ is integration constant, $H_{0}$ is the so-called 'differential constant', and $D_{x}^{m}, D_{t}^{n}$ are Hirota operators [8] (See Appendix A). If in (9) we choose $\beta_{1}=12 \alpha$ and $H=-\left(H_{0}+\frac{\beta_{0}^{2}}{24 \alpha}\right) / 4$, then the bilinear equation (9) can be represented as a conjunction from the two equations, as follows:

$$
\begin{gathered}
\left(D_{t} D_{x}+\lambda D_{x}^{4}+8 G\right) \zeta . \zeta=0, \\
{\left[\left(1+\beta_{0}\right) D_{x}^{2}+\alpha D_{x}^{4}-8 H\right] \zeta . \zeta=0 .}
\end{gathered}
$$

We can draw the conclusion that for $\alpha_{2}=\alpha_{3}$ KVE is a semi-integrable equation, since both residual equations (10) and (11) have a bidifferential structure [9], [10] and [11]. We will search for the solution of the last two equations 
in the form [12]:

$$
\zeta(\xi)=\theta_{4}(\xi, q)=\sum_{n=-\infty}^{\infty}(-1)^{n} q^{n^{2}} e^{2 i n \xi}, \xi=k x+\omega t+\delta,
$$

where $\theta_{4}(\xi, q)$ is the fourth Jacobi theta function [6], which is well defined for every $\xi$, if $0<|q|<1$. By substituting $\zeta(\xi)$ from (12) into (10) and (11), and by making use of the identity [8]

$$
D_{x}^{m} e^{k_{1} x} \cdot e^{k_{2} x}=\left(k_{1}-k_{2}\right)^{m} e^{\left(k_{1}+k_{2}\right)^{x}}, \quad m \in \mathbb{N}, k_{1} \neq k_{2},
$$

we will obtain the infinite systems

$$
\sum_{m=-\infty}^{\infty} F_{1}(m) e^{2 i m \xi}=0 ; \quad \sum_{m=-\infty}^{\infty} F_{2}(m) e^{2 i m \xi}=0
$$

where

$$
\begin{gathered}
F_{1}(m)=\sum_{n=-\infty}^{\infty}\left[-4 k \omega(2 n-m)^{2}+16 \lambda k^{4}(2 n-m)^{4}-8 G\right] q^{n^{2}+(n-m)^{2}} ; \\
F_{2}(m)=\sum_{n=-\infty}^{\infty}\left[-4 k^{2}\left(1+\beta_{0}\right)(2 n-m)^{2}+16 \alpha k^{4}(2 n-m)^{4}-8 H\right] q^{n^{2}+(n-m)^{2}} .
\end{gathered}
$$

The bilinear structure of (10) and (11) [9], [10] allows us to apply the index parity principle to (13) [10], [11], thus obtaining their compact forms

$$
F_{j}(0) \theta_{3}\left(2 \xi, q^{2}\right)-q^{\frac{1}{2}} F_{j}(1) \theta_{2}\left(2 \xi, q^{2}\right)=0, \quad j=1,2
$$

where $\theta_{j}\left(2 \xi, q^{2}\right), j=2,3$ are the second and third Jacobi theta functions [6]. The last equality yields the four equations

$$
F_{1}(0)=F_{1}(1)=0, F_{2}(0)=F_{2}(1)=0,
$$

which are easily reduced to the linear algebraic systems (See Appendix B):

$$
\begin{aligned}
& k q \theta_{3}^{\prime} \omega+\theta_{3} G=8 \lambda k^{4} q\left(\theta_{3}^{\prime}+q \theta_{3}^{\prime \prime}\right) ; \quad\left(1+\beta_{0}\right) k^{2} q \theta_{3}^{\prime}+\theta_{3} H=8 \alpha q k^{4}\left(\theta_{3}^{\prime}+q \theta_{3}^{\prime \prime}\right) ; \\
& k q \theta_{2}^{\prime} \omega+\theta_{2} G=8 \lambda k^{4} q\left(\theta_{2}^{\prime}+q \theta_{2}^{\prime \prime}\right) ; \quad\left(1+\beta_{0}\right) k^{2} q \theta_{2}^{\prime}+\theta_{2} H=8 \alpha q k^{4}\left(\theta_{2}^{\prime}+q \theta_{2}^{\prime \prime}\right),
\end{aligned}
$$


where $\theta_{j}=\theta_{j}\left(0, q^{2}\right), j=2,3$. Both algebraic systems are compatible and definite because if $\Delta_{j}(k, q), j=1,2$ are their determinants, then

$$
\Delta_{2}(k, q)=k \Delta_{1}(k, q)=k^{2} q\left(\theta_{2} \theta_{3}^{\prime}-\theta_{2}^{\prime} \theta_{3}\right)=k^{2} q W\left(\theta_{2}, \theta_{3}\right) \neq 0,
$$

where $W\left(\theta_{2}, \theta_{3}\right)$ is the Wronskian from the linearly independent functions $\theta_{2}\left(0, q^{2}\right)$ and $\theta_{3}\left(0, q^{2}\right)$. The solutions of this system are, as follows:

$$
\begin{gathered}
\omega(k, q)=8 \lambda k^{3}\left[1+q \frac{W^{\prime}\left(\theta_{2}, \theta_{3}\right)}{W\left(\theta_{2}, \theta_{3}\right)}\right] ; \quad G(k, q)=8 \lambda k^{4} q^{2} \frac{W\left(\theta_{2}^{\prime}, \theta_{3}^{\prime}\right)}{W\left(\theta_{2}, \theta_{3}\right)} \\
\beta_{0}(k, q)=\frac{\alpha \omega(k, q)}{\lambda k}-1 ; \quad H(k, q)=\frac{\alpha}{\lambda} G(k, q) .
\end{gathered}
$$

For the admissible values of the wave number $(k>0)$ and the admissible values of the perturbation parameter $q(0<|q|<1)$, considering the solutions (15) and (16), we obtain the complex solution

$$
u(x, t)=\left(\frac{\alpha \omega(k, q)}{\lambda k}-1\right)+12 \alpha \frac{\partial^{2}}{\partial x^{2}}\left[\ln \theta_{4}(\xi, q)\right] .
$$

In the general case, $(17)$ is a localized periodic solution of $\operatorname{KVE}(\mu=1)$, which is a complex meromorphic function with double poles at the lattice

$$
\xi_{m n}=m+i(n+1 / 2) \operatorname{Im}(\tau), \quad q=e^{i \pi \tau}, \quad \operatorname{Im}(\tau)>0, \quad m, n \in \mathbb{Z} .
$$

We will choose the dynamic parameters $k, q, \delta$ so that the periodic solution (17) would be real, bearing in mind the applicability from physical point of view. Let us set:

$$
\tau=i \varepsilon, \quad \varepsilon>0, \text { i.e. } q=e^{-\pi \varepsilon}<1
$$

If under the condition of hypothesis (18), we impose the limitation for the phase variable to take values in the horizontal stripe:

$$
-\pi \varepsilon<\operatorname{Im}(\xi)<\pi \varepsilon
$$

then in the localized solution (17) we will isolate the singularities generated by the double poles $\xi_{m n}$. In fact, this horizontal stripe can be considered as a domain of analyticity of (17). 
In a 'small amplitude' regime, i.e. $\varepsilon \rightarrow 0(q \rightarrow 1)$, the meromorphic solution (17) generates a superposition of sinusoidal harmonics, resulting from the Fourier representation of the logarithmic derivative

$$
\frac{\theta_{4}^{\prime}(\xi, q)}{\theta_{4}(\xi, q)}=4 \sum_{m=1}^{\infty} \frac{q^{m}}{1-q^{2 m}} \sin 2 m \xi=2 \sum_{m=1}^{\infty} \operatorname{cosech}(\varepsilon \pi m) \sin (2 m z) .
$$

As a result we obtain the periodic sinusoidal solution

$$
u(x, t)=\left(\frac{\alpha \omega}{k \lambda}-1\right)+24 \alpha k^{2} \sum_{m=-\infty}^{\infty} m \operatorname{cosech}(\varepsilon \pi m) \cos (2 m \xi),
$$

where $\omega(k, \varepsilon)$ is as in (15) and $q=e^{-\varepsilon \pi}$. To obtain validity of the Fourier representation in the strongly non-linear zones as well, i.e. for $\varepsilon \rightarrow \infty(q \rightarrow 0)$, it is suitable to define a new perturbation parameter $q_{0}=e^{i \pi \tau_{0}}$, with $\tau_{0}=-1 / \tau$, and hence the boundary transition $q \rightarrow 1$ is equivalent to the transition $q_{0} \rightarrow 0$. From the quasi-periodic transformation (of first degree) for $\theta_{4}[6]$

$$
\theta_{4}(\xi, q)=\left(-i \tau_{0}\right)^{1 / 2} e^{i \tau_{0} \xi^{2} / \tau} \theta_{2}\left(\tau_{0} \xi, q_{0}\right),
$$

together with the formula [6]

$$
\frac{d^{2}}{d z^{2}}\left[\ln \theta_{2}(z, q)\right]=-\sum_{m=-\infty}^{\infty} \sec h^{2}[i(z-\tau \pi m)]
$$

we can reduce the meromorphic solution (17) into a superposition of periodic solitary-wave forms

$$
u(x, t)=\left(\frac{\alpha \omega}{k \lambda}-1\right)+12 \alpha k^{2} \sum_{m=-\infty}^{\infty} \sec h^{2}\left(\frac{\xi-m \pi}{\varepsilon}\right) .
$$

\section{Conclusion}

The role of the perturbation parameter $q$ is to cause transition phenomena, both in the case of integrable partial differential equations and semiintegrable equations (as is the case of $\operatorname{KVE}\left(\alpha_{2}=\alpha_{3}\right)$ ). When we choose $q=e^{-\varepsilon \pi}$, where $\varepsilon>0$, then for $\varepsilon \rightarrow 0$ (i.e. $q \rightarrow 1$ ), wide overlap zones are formed in the adjacent solitary wave forms, and a wave localization process sets in. Non-linear sinusoidal wave packages take shape, whose dynamics is 
described by (20). This wave scheme is close to the linear one, which is characterized by the fact that the non-linear effects are weak. Under the boundary transition $\varepsilon \rightarrow \infty$ (i.e. $q \rightarrow 0$ ), a transition towards separation and differentiation of these forms starts in the adjacent solitary wave forms, resulting from the increasingly growing wave length. The overlap zones are on the decrease generating delocalization of the wave forms. The evolution of these solitary-wave forms is described by means of (21). There, the non-linear effects are quite evident. It is worth noting a characteristic feature of the obtained periodic solutions (20), (21). Their spatial shifts:

$$
\alpha(k, q)=\frac{\alpha \omega(k, q)}{k \lambda}-1
$$

are fixed for specific values of the parameters $k, q$ for each harmonics. This circumstance contrasts with the spatial shifts of the non-integrable partial differential equations ([9], [10], [11]).

\section{Appendix A. Logarithmic derivatives expressed by the $\mathrm{Hi}$ - rota's operators $D_{t}, D_{x}$}

$$
\left\{\begin{array}{l}
(\ln \varphi)_{x x}=\frac{D_{x}^{2} \varphi \cdot \varphi}{2 \varphi^{2}} ; \\
(\ln \varphi)_{x x x}=\frac{\varphi_{x x x}}{\varphi}-3 \frac{\varphi_{x} \varphi_{x x}}{\varphi^{2}}+2 \frac{\varphi_{x}^{3}}{\varphi^{3}} ; \\
(\ln \varphi)_{x x x x}=\frac{D_{x}^{4} \varphi \cdot \varphi}{2 \varphi^{2}}-6\left(\frac{D_{x}^{2} \varphi \cdot \varphi}{2 \varphi^{2}}\right)^{2} ; \\
(\ln \varphi)_{t x x x}=\frac{D_{t} D_{x}^{3} \varphi \cdot \varphi}{2 \varphi^{2}}-6\left(\frac{D_{t} D_{x} \varphi \cdot \varphi}{2 \varphi^{2}}\right)\left(\frac{D_{x}^{2} \varphi \cdot \varphi}{2 \varphi^{2}}\right) ; \\
(\ln \varphi)_{x x x x x x}=\frac{D_{x}^{6} \varphi \cdot \varphi}{2 \varphi^{2}}-30\left(\frac{D_{x}^{2} \varphi \cdot \varphi}{2 \varphi^{2}}\right)\left(\frac{D_{x}^{4} \varphi \cdot \varphi}{2 \varphi^{2}}\right)+120\left(\frac{D_{x}^{2} \varphi \cdot \varphi}{2 \varphi^{2}}\right)^{3}
\end{array}\right.
$$


Appendix B. Identities with the Jacobi theta functions $\theta_{3}, \theta_{2}$

$$
\left\{\begin{array}{l}
\sum_{n=-\infty}^{\infty} q^{2 n^{2}}=\theta_{3} ; \sum_{n=-\infty}^{\infty} q^{n^{2}+(n-1)^{2}}=q^{1 / 2} \theta_{2} \\
\sum_{n=-\infty}^{\infty} n^{2} q^{2 n^{2}}=q \theta_{3}^{\prime} / 2 ; \sum_{n=-\infty}^{\infty}(2 n-1)^{2} q^{n^{2}+(n-1)^{2}}=2 q^{3 / 2} \theta_{2}^{\prime} \\
\sum_{n=-\infty}^{\infty} n^{4} q^{2 n^{2}}=q\left(\theta_{3}^{\prime}+q \theta_{3}^{\prime \prime}\right) / 4 ; \sum_{n=-\infty}^{\infty}(2 n-1)^{4} q^{n^{2}+(n-1)^{2}}=4 q^{3 / 2}\left(\theta_{2}^{\prime}+q \theta_{2}^{\prime \prime}\right) \\
\sum_{n=-\infty}^{\infty} n^{6} q^{2 n^{2}}=q\left(\theta_{3}^{\prime}+3 q \theta_{3}^{\prime \prime}+q^{2} \theta_{3}^{\prime \prime \prime}\right) / 8 \\
\sum_{n=-\infty}^{\infty}(2 n-1)^{6} q^{n^{2}+(n-1)^{2}}=8 q^{3 / 2}\left(\theta_{2}^{\prime}+3 q \theta_{2}^{\prime \prime}+q^{2} \theta_{2}^{\prime \prime \prime}\right)
\end{array}\right.
$$

\section{REFERENCES}

[1] Topper, J., T. Kawahara. Approximate Equations for Long Nonlinear Waves on a Viscous Fluid. J. Phys. Soc. Japan, 44 (1978), 663-666.

[2] Cohen, B. I., J. A. Krommes, W. M. Tang, M. N. Rosenbluth. Nonlinear saturation of the dissipative trapped-ion mode by mode coupling. Nuclear Fusion, 16 (1976), 333-335.

[3] Garcia-Ybarra P. L., J. L. Castillo, M. G. Velarde. A Nonlinear Evolution Equation for Benard-Marangoni Convection with Deformable Boundary. Phys. Lett. A, 122 (1987), 107-110.

[4] Garcia-Ybarra P. L., J. L. Castillo, M. G. Velarde. Benard-Marangoni Convection with a Deformable Interface and Poorly Conducting Boundaries. Physics Fluids, 30 (1987), 2655-2661.

[5] Hyman J. M., B. Nicolaenko. Coherence and Chaos in the Kuramoto-Velarde Equation, in Directions in Partial Differential Equations, New York, Academic Press, 1987.

[6] Lowden, D. F. Elliptic Functions and Applications, Berlin, Springer, 1989.

[7] Hirota, R., J. Satsuma. A Variety of Nonlinear Network Equations Generated from the Bäcklund Transformations for the Toda Lattice. Prog. Theoret. Phys. Suppl., 59 (1976), 64-100. 
[8] Hirota, R. Exact N-solution of the Wave Equation of Long Waves in ShallowWater in Nonlinear Lattices. J. Math. Phys., 14 (1973), 810-814.

[9] Kamenov, O. Y. Exact Periodic Solutions of the Sixth-Order Generalized Boussinesq Equation. J. Phys. A: Math. Theor., 42 (2009), 375501.

[10] Kamenov, O. Y. Periodic Solutions of the Non-Integrable Convective Fluid Equation. J. Math. Phys., 53 (2012), 063705.

[11] Kamenov, O. Y., A. P. Angova. Exact Periodic Solutions of the Nonintegrable Kawahara Equation. International Scholarly Research Network (ISRN) Mathematical Physics, (2012), 185469.

[12] Parker, A. Periodic Solutions of the Intermediate Long-wave Equation: a Nonlinear Superposition Principle. J. Phys. A: Math. Gen., 25 (1992), 2003-2032. 